\title{
Estimating the Size of the Methamphetamine-Using Population in New York City Using Network Sampling Techniques
}

\author{
Kirk Dombrowski ${ }^{1 *}$, Bilal Khan ${ }^{1}$, Travis Wendel ${ }^{1}$, Katherine McLean $^{2}$, Evan Misshula ${ }^{2}$, \\ Ric Curtis ${ }^{1}$ \\ ${ }^{1}$ Social Networks Research Group, John Jay College, CUNY, New York, USA \\ ${ }^{2}$ CUNY Graduate Center, New York, USA \\ Email: "kdombrowski@jjay.cuny.edu
}

Received July 21 ${ }^{\text {st }}, 2012$; revised August $24^{\text {th }}, 2012$; accepted September $10^{\text {th }}, 2012$

\begin{abstract}
As part of a recent study of the dynamics of the retail market for methamphetamine use in New York City, we used network sampling methods to estimate the size of the total networked population. This process involved sampling from respondents' list of co-use contacts, which in turn became the basis for capture-recapture estimation. Recapture sampling was based on links to other respondents derived from demographic and "telefunken" matching procedures-the latter being an anonymized version of telephone number matching. This paper describes the matching process used to discover the links between the solicited contacts and project respondents, the capture-recapture calculation, the estimation of "false matches", and the development of confidence intervals for the final population estimates. A final population of 12,229 was estimated, with a range of $8235-23,750$. The techniques described here have the special virtue of deriving an estimate for a hidden population while retaining respondent anonymity and the anonymity of network alters, but likely require larger sample size than the 132 persons interviewed to attain acceptable confidence levels for the estimate.
\end{abstract}

Keywords: Population Estimation; Network Methods; Methamphetamine; Anonymous Sampling

\section{Introduction}

Statistics such as the size of hard-to-enumerate populations are both important and difficult challenges for social science: important in that they represent one area where sociological results impact the allocation of public funds for both law enforcement and public health resources (Aceijas et al., 2006, Dengenhardt \& Hall, 2012), yet difficult because they often require estimation procedures that pit ideal methods against the difficulties of research implementation. Such questions lie at the heart of applied sociology. In particular, estimates of the size of hidden populations often hinge on data drawn from a single source, such as arrests or hospital admissions, whose relationship to overall population levels remains largely unknown, leaving both policy makers and researchers unsatisfied with results. Recent modeling work not withstanding (Simeone et al., 2003; Zhao, 2011; see Berchenko \& Frost, 2011 for discussion) this represents a less than ideal situation, a point aptly summed up in the titled of a recent article: "The numbers game: Let's all guess the size of the illegal drug industry!" (Thoumi, 2005). As noted by Thoumi, such problems are particularly true for drug using populations, where limited data from disparate sources often indicates countervailing trends, yet population estimates and overall community dynamics continue to occupy important policy decisions. In these situations, research confronts hidden populations whose illegal behaviors invoke the need for anonymous sampling, further exacerbating an already difficult research scenario.

New York City methamphetamine users represent such a population. Indeed, meth-users in NYC have received little

${ }^{*}$ Corresponding author. attention until recently when concern about growing levels of methamphetamine use were associated HIV risk behaviors in the MSM (men who have sex with men)/gay community (Hirshfield et al., 2004; Morin et al., 2005). Methamphetamine has actually been available in New York City for decades (Drug Enforcement Administration (DEA) 2004, 2006, National Drug Intelligence Center (NDIC) 2008). Yet New York's methamphetamine markets have remained mostly inaccessible to researchers, and the small body of literature that is currently available on methamphetamine use in New York City focuses mainly on use among MSM while offering little information about market size, numbers of users, or distribution in general; nor about use outside of MSM communities, and what effect this has on the total number of users in the area. Local data such as these are important. While DAWN (2009: pp. 18-19) reports that the national estimate of methamphetamine-related emergency room visits in the US dropped from 132,576 in 2004 to 66,308 in 2008, and ADAM II (2009) data show significant declines in those testing positive for methamphetamine upon arrest, the NDIC (2008) notes that "the number of amphetamine-related (including methamphetamine-related) admissions to publicly funded treatment facilities in the New York/New Jersey Region increased 15 percent overall from 2002 (685) to 2006 (787)".

\section{Network-Based Population Estimates}

Estimation techniques for hidden population sizes using social network techniques have grown as sociological exposure to social network analysis has exploded over the last two decades. Among the most popular of these techniques is Respondent 
Driven Sampling developed by sociologist Douglas Heckathorn (1997, 2002, 2007; see recent review of 128 RDS studies by Johnston et al., 2008). However, RDS does not present overall population sizes (rather, only population prevalences) and has recently received some criticism for its base estimation procedures (see Gile et al., 2012 for a summary of those criticisms). Handcock and Giles' proposed replacement estimator (the "sequential sampling" estimator, see Gile \& Handcock, 2010) relies, however, on an estimate of the total size of the hidden population - and thus reintroduces a variable that the original RDS estimators had sought to escape. Given the adoption of RDS estimation by the World Health Organization (for estimating national rates of HIV and AIDS) and UNAIDS, and growing interest in using network techniques in determining overall size estimations of hidden populations, in this paper we propose a method of network-based capture-recapture population estimation that involves only a single sampling round (rather than the two rounds implied by standard capture-recapture techniques) and which can be used to supplement RDS data collection or more conventional venue based approaches.

The method proposed below is capable of producing total population estimates which can be used with the Gile and Handcock estimator, or as a means for supplementing the original RDS estimator with a total population estimate for the group in question. And perhaps most importantly, it does so while maintaining respondent anonymity, a crucial consideration when dealing with drug using and other illegal or highly stigmatized behaviors. This factor, taken together with the fact that the recapture phase takes place simultaneous with the original capture phase of the sampling, and the easy fit of the technique with ordinary RDS methods, creates what we feel to be an important new tool for applied sociological research. To show an application of the technique in concrete terms, we demonstrate the development of an estimate for the population of methamphetamine users in New York City.

This method contrasts with two other network-related attempts to estimate total population size: 1) network scale-up methods and 2) other capture-recapture methods using multiple RDS samples. In the words of a recent summary, network scale-up methods (or NSUM) "rests on the assumption that people's social networks - the set of people whom you 'know' - are, on average, representative of the general population in which you live and move" (Bernard et al., 2010: p. ii12). In this procedure, individual estimates of sub-populations are "scaled" to aggregate levels, and the estimates of many individuals are combined. For example, if a respondent answers that he/she knows two pregnant women out of a total of 100 contacts, we could estimate the number of pregnant women in his/her county of 10,000 people (via consistent proportion) to be 200. By combining this estimate with the estimates drawn from many others, more accurate figures can be obtained. NSUM advocates see this as a means for estimating the size of sub-populations that may be known but difficult to enumerate. Still, significant problems arise for NSUM methods when trying to estimate rates of participation in activities that individuals might try to keep secret even (or especially) from close associates (see Salganik et al., 2011). Such a situation, obviously, could occur with any illegal or highly stigmatized activity, such as illegal drug use.
A second popular method of estimation depends less on information known to individuals and more on researchers ability to reach hidden populations repeatedly (by means, for example, such as successive waves of Respondent Driven Sampling). According to the logic of capture-recapture studies, successive samples that discover a proportion of identical individuals can be used to estimate the total population size by the well-known Lincoln-Peterson formula (discussed below). Multiple resampling increases the accuracy of these predictions. Where RDS has proven capable of reaching large samples of hidden populations, it would appear ideally suited to such tasks. Problems arise, however, where initial sampling paths can be seen to affect subsequent referral paths, thus skewing the "recapture" process to those in the original sample (and resulting in an inaccurate recapture number, see Berchenko \& Frost, 2011). Given these issues, what seems needed is a process that is less susceptible to discovery bias around stigmatized behaviors (a problem for NSUM) and not dependent on resampling procedures that may be biased by initial sampling (as is the issue for RDS-based capture-recapture methods), and finally, one that is capable of retaining respondent anonymity throughout the research process. Below we propose such a method.

\section{Estimating the Size of the NYC Methamphetamine Using Population}

In an attempt to estimate the size of the New York City methamphetamine using population, we have developed a network-based variant of standard capture-recapture methods that is capable of estimating the total size of a hidden, networked population from a network sample of current users, even while maintaining respondent anonymity. The proposed method requires sampling from each respondent's network connections, and matching these connections against both the other respondents in the sample and the list of their respective contacts. Such methods are not particularly complex, and make use of capture/recapture methods with a long history in both social and biological sciences. In current circumstances, however, considerable modifications are required, as network sampling in the context of illicit and often socially stigmatized activity requires retaining anonymity of both research subjects and their network connections. These concerns necessarily complicate the matching of contacts assumed by the capture-recapture methods. For this reason, a naïve matching strategy of simply matching the names of respondents and contacts across interviews is not possible. We address this challenge by a novel means of establishing network connections while maintaining the anonymity of participants and their contacts which we refer to as the "telefunken method".

This process requires the recruitment of a sample pool of network participants and the elicitation of a number of contacts from each. In addition to personal descriptives later used in the matching process, each participant was asked for his/her own "telefunken code", derived from the last three digits of their own mobile phone number. To arrive at the code, each of the three digits is encoded as being either even or odd, and low or high (with 4.5 being the threshold). Together with height, approximate weight, hair color, eye color, gender, and race/ethnicity, this produced a six bit code for each respondent that served in matching the respondent to contacts reported by other 
study respondents ${ }^{1}$. Importantly, the telefunken encoding ensures (and assures) that actual telephone numbers of respondents remain unknown to researchers throughout the study. As will be seen below, a critical question raised by this method is the estimation of error scores (in the event of false matches between individuals who by coincidence have the same code) and error estimation of the resulting population estimate. We note that these questions would be greatly simplified by attaining a code for more phone number digits. In our case, however, pre-testing found that asking for more than 3 digits raised suspicion among our research subjects and equally importantly, questions about the assurance of anonymity by our Institutional Review Board. Given these concerns, a method capable of producing and bounding an estimate within a range of confidence estimates seems particularly important.

In the current study, respondents were recruited using Respondent Driven Sampling (RDS), an established research method for anonymously recruiting hard-to-reach populations (Heckathorn, 1997, 2002, 2007) such as the New York City methamphetamine user network. This process resulted in the recruitment of 132 eligible participants, starting from $(n=37)$ RDS "seeds" reached using a Craigslist advertisement. Additional $(n=95)$ respondents were obtained by referrals via the standard RDS protocol. Respondent interviews included a number of use-related questions, and the appearance-based and demographic information. Further, in addition to their own personal information and telefunken code, each respondent was asked to select up to five methamphetamine-using contacts whose phone number they currently had in their mobile phone's directory. This selection was carried out by choosing initial letters of last names from a randomized list of alphabet letters ${ }^{2}$. The respondent was then questioned about the randomly selected contacts, in order to obtain data on the contacts' personal characteristics (approximate height, approximate weight, hair color, eye color, gender, and race/ethnicity) and telefunken code.

For purposes of the population estimate, project respondents were treated as the "capture" population, while each of the contacts provided during the interviews ("reports") was considered a "recapture assay". By finding the number of original respondents discovered via recapture assays (as a proportion of the total number of assays), researchers had a basis for estimateing the overall size of the population under consideration. Again, among the main contribution of the proposed method is that anonymity can be maintained throughout the process, with personal descriptions and telefunken codes together forming the sole means of identification and matching.

Capture-recapture methods have been used extensively in estimating population levels in biology and epidemiology, and more recently, employed in conjunction with methods designed to sample hidden populations of people (Bouchard, 2007; Hope et al., 2005; Paz-Bailey et al., 2011). At issue in these approaches is not normally the validity of the standard Lincoln-Peterson methodology or its appropriateness to the problem, but rather the question of whether the original "capture" or

${ }^{1}$ For example, the telefunken code for any phone numbers which end in 123 (or 343, or 301) is odd-even-odd-low-low-low, while for phone numbers ending in 701 (or 523) the code is odd-even-odd-high-low-low. The name "telefunken" is borrowed from a Frank Zappa song (from the album Joe's Garage). It is intended to imply "funky telephone" code, as we felt like this was a good description of the coding method used here.

${ }^{2}$ Those respondents with five or fewer use-contacts in their mobile phone directory simply selected all of them without using the randomized alphabet page. subsequent "recapture" techniques are, in fact, sufficiently random (see Berchenko \& Frost, 2011 for review and discussion). This issue is taken up in the discussion, below, but we note here that one difference between past studies and the method described here is that this method does not depend on data from outside the study (such as arrest numbers or hospital admissions) to determine either the capture or recapture statistic. Both are determined simultaneously during the sampling/recruitment process. Whether this results in an advantage or disadvantage over capture-recapture methods dependent on external data sources likely depends on context. Regardless, in this sense the proposed strategy represents a significant departure from other uses of capture-recapture in drug use and other research.

The remainder of this paper details the steps involved in two separate attempts to estimate the methamphetamine using population in New York City ${ }^{3}$. As will be seen below, an estimate from the joint population was required due to the small sample size of the research population. Even with this second step, the range of estimates is still quite wide. One may conclude from this fact that the current method leaves much to be desired. The "cup half full" interpretation, however, is that the current method is able to produce a statistically sound method for population estimation of a hidden population from a relatively small sample, and to do so while maintaining anonymity. It is this fact that, we feel, makes this method an important new tool in research on illegal activities where questions of anonymity and the protection of human subjects are paramount.

\section{Baseline Estimate}

The population estimate $(P)$ entails a capture/recapture form of estimation using the respondents $(n=132)$ to define the capture population, and matches between the reports $(s=466)$ and the respondents to define the recaptured subset. Matches are defined by considering seven categorical variables: telefunken code, gender, race, height, weight, hair color, and eye color. A respondent from the original sample was said to "match" a report if the two agreed on all seven of these variables. With this definition, we found there were 11 matches between the 466 reports and the 132 respondents ${ }^{4}$. These 11 matches were used to define the recapture number $(t=11)$. Naïve extrapolation from this capture/recapture paradigm using the Lincoln-Peterson method yields:

$$
P=\frac{n \cdot s}{t}
$$

where $P$ is the total estimated population, $n$ is the size of the capture population, $t$ is the recapture number, and $s$ is the number of recapture assays. Using 11 matches between 466 reports, and an initial sample of 132 respondents, yields a population estimate $P=5592$. The sections that follow provide successive refinements to this figure.

\footnotetext{
${ }^{3}$ The choice of NYC was not arbitrary. We received a grant to do a population estimate of methamphetamine users in New York City (among other things) from the US National Institute of Justice, and so the necessary data was collected there. No similar data is available for a similar population in another city for comparative purposes, nor are other formal estimates for the size of the NYC meth using population available via other methods. This significantly limits the comparability of the results and the opportunities for their verification by other means, though we hope this will be remedied in the future.

${ }^{4}$ The details of the matching procedure, which utilized approximate matching of height, weight, and other continuous variables, is described in the appendix of the original project report (Wendel et al., 2011).
} 


\section{False Matches}

The matching technique maintains anonymity of both respondents and reports by considering general characteristics that are shared by entire segments of the ambient population of methamphetamine users, but the technique also introduces the possibility of "false matches" during the matching process. In particular, a false match occurs whenever a report "matches" a respondent based on agreement across all seven criteria, but when the report actually refers to someone outside of our sample. Indeed, because false matches are possible, we have possibly over-estimated the recapture number $(t=11)$, and hence the $P=5592$ estimate should be taken as a conservative lower estimate of population size.

To further refine the population estimate, it is necessary to consider the probability distribution governing the number of matches (amongst the 11 telefunken matches observed) that are likely to be "false".

\section{Initial Estimation via Marginals}

To estimate the expected number of false matches $E[F]$, we shall need to refer to the marginal sample distributions of each categorical variable involved in the matching process (see Table 1). We assume that the sample size is large enough so that its marginals approximate the population marginals. In addition, in this first attempt at refining the population estimate, we assume that the six categorical variables are independent. We begin by way of illustrative example. Consider a categorical variable $V$, say Gender. The possible values assumed by $V$ are known:

$$
\left\{x_{1}=\text { Male, } x_{2}=\text { Female, } x_{3}=\text { Transgender }\right\}
$$

and associated probabilities are computable from the marginals in Table 1:

$$
\begin{aligned}
& \operatorname{Prob}(V=\text { Male })=119 / 132 \\
& \operatorname{Prob}(V=\text { Female })=11 / 132 \\
& \operatorname{Prob}(V=\text { Transgender })=2 / 132 .
\end{aligned}
$$

Suppose we choose two individuals at random from an infinite population satisfying the above marginal distribution for the Gender variable. Since 119 of the 132 respondents were male, the probability that both individuals in this pair will be male is $(119 / 132)^{2}$, or 0.81 (i.e. about $81 \%$ of the time). Similarly, the probability of the two individuals both being female is $(11 / 132)^{2}=0.007$, or about $0.7 \%$ of the time. Finally, the probability of the individuals both being transgender is $(2 / 132)^{2}$ $=0.0002$, a mere $0.02 \%$ of the time. The total probability of a match across the Gender variable is then given by:

$$
(119 / 132)^{2}+(11 / 132)^{2}+(2 / 132)^{2}=0.82 .
$$

Repeating this same calculation we can determine the probability of agreement between the two individuals for each of the other variables (race, gender, hair color, eye color, height and weight). The results are shown in Table 2. Now, assuming independent sequential assignment of categorical variables, the probability that two randomly chosen individuals will match on all six descriptive categorical variables is the product of the individual probabilities listed in Table 2:

$0.3805 \times 0.8198 \times 0.3257 \times 0.7072 \times 0.2320 \times 0.2236=3.72 \times 10^{-3}$

Since each telefunken code is 6 bits, there are $2^{6}=64$ distinct codes, and thus, the probability that two individuals will match by sheer chance, is given by:
Table 1.

Sample distributions by attribute values.

\begin{tabular}{cl}
\hline $\begin{array}{c}\text { Attribute } \\
(k)\end{array}$ & \multicolumn{1}{c}{ Category $(n=)$} \\
\hline Race (5) & $\begin{array}{l}\text { Black/African American (71) Hispanic (26) White (30) } \\
\text { Asian (3) Other (2) }\end{array}$ \\
Gender (3) & Male (119) Female (11) Trans (2) \\
Hair (5) & $\begin{array}{l}\text { Black (65) Brown (21) Blonde (3) Grey/Salt and Pepper } \\
\text { (10) Other (30) }\end{array}$ \\
Eye (2) & Brown/Dark (109) Blue/Green/Light (21) \\
Height (5) & $\begin{array}{l}\text { Below 5'4”, (8) 5'4”-5'8”, (36) 5'7’'-5'11” (24) Over 6'1” (9) } \\
\text { 5'10) }\end{array}$ \\
Weight (5) & $\begin{array}{l}\text { Below 125-145 (15) 135-165 (41) 155-185 (36) 175-205 } \\
\text { (23) Over 195 (13) }\end{array}$ \\
\hline
\end{tabular}

Table 2.

Probability of agreement between randomly selected sample members by attributes.

\begin{tabular}{lcc}
\hline Attribute & Sum of the Squares of the Marginals & $\begin{array}{c}\text { Probability of } \\
\text { Agreement }\end{array}$ \\
\hline Race & $0.2893+0.0388+0.0517+0.0005+0.0002$ & 0.3805 \\
Gender & $0.8127+0.0069+0.0002$ & 0.8198 \\
Hair Color & $0.2425+0.0253+0.0005+0.0057+0.0517$ & 0.3257 \\
Eye Color & $0.6819+0.0253$ & 0.7072 \\
Height & $0.0037+0.0744+0.1162+0.0331+0.0046$ & 0.2320 \\
Weight & $0.0129+0.0964+0.0743+0.0303+0.0097$ & 0.2236 \\
\hline
\end{tabular}

$$
3.72 \times 10^{-3} \times 1 / 64=5.81 \times 10^{-5} .
$$

For any specific respondent then, the expected the number of reports (drawn from a population represented accurately by the sample itself) that would telefunken match by sheer chance is:

$$
466 \times\left(5.81 \times 10^{-5}\right)=2.71 \times 10^{-2} \text {. }
$$

The expected total number of false matches over all $(n=132)$ respondents can now be estimated using linearity of expectation:

$$
F^{\prime}=132 \times\left(2.71 \times 10^{-2}\right)=3.58
$$

The number $F^{\prime}=3.58$ provides an initial estimate of $E[F] \approx$ $F^{\prime}$ which takes into account the marginal distributions of the population from which the sample is drawn (to the extent that the marginals of the population conform to those of the sample). Adjusting the recapture number $t^{\prime}=t-F^{\prime}$ to incorporate these findings yields $t^{\prime}=11-3.58=7.42$ and the revised population estimate $P^{\prime}=8290$.

\section{Better Estimate via the Joint}

The previous estimate of false matches provided a first attempt at correcting for the fact that the number of matches generally exceeds the true recapture set. Nonetheless, there are some shortcomings to the false match estimation procedure described above. In particular, the procedure outlined above 
assumed independent assignment of categorical variables, where in actuality our sample did not always reflect this assumption, since several variables were clearly not independent (e.g. height and weight). In more formal terms, the joint probability of randomly finding someone of African American ethnicity with blond hair, for example, was not well-estimated by the product of probabilities specified in the marginal distributions of ethnicity and hair color. Indeed, the only property that one could safely assume to be independent of all others is the telefunken code.

One approach to the problem of non-independence would be to establish the relationships among the six attributes used in the matching process. However, quantifying the dependencies between the six variables would be daunting. Instead, we chose to consider all six variables simultaneously using a single joint distribution across all possible combinations of their values. Such an approach presented its own difficulties, however. To describe these issues, it is helpful to define the notion of a class to be a six-tuple of attribute values (one value for each of the six variables). Let $C$ denote the set of distinct classes that might be manifested by study respondents. Examining the categories listed in Table 1, we see that:

$$
|C|=5 \times 3 \times 5 \times 2 \times 5 \times 5=3750 \text { classes. }
$$

Although 3750 classes were potentially possible, only 128 classes were actually manifested by the $(n=132)$ sampled respondents. Thus, the sample provided very little information about the relative likelihoods of classes under the joint distribution, since the sample distribution over $C$ was either 0 or $1 / 132$ across almost all classes. The source of this difficulty was due to having too small a sample to effectively model the joint distribution, and was this addressed by the best-case-available remedy of adding the $(s=466)$ reports to the $(n=132)$ sample to obtain a larger "extended sample" of 598 individuals. When the joint distribution was estimated using this extended sample, it was found to manifest non-zero probabilities for 290 distinct classes in $C$ with broad variations in probability mass. For example, two classes exhibiting non-zero probability were:

Hispanic, male, black hair, brown eyes, 5'4"-5' 8", 135-165lbs (One of the $(\mathrm{n}=132)$ respondents exhibited these characteristics)

and

Black, female, black hair, brown eyes, 5'7'-5'11', $155-185 \mathrm{lbs}$ (One of the $(\mathrm{s}=466)$ reports exhibited these characteristics).

Restated more formally, the joint distribution is defined over the set of classes $c_{i}$ in $C$, and the joint probability of an individual belonging to class $c_{i}$, denoted $p\left(c_{i}\right)$, can be estimated using the proportion of individuals in the extended sample that were found to belong to class $c_{i}$. To the extent that the distribution

$$
\left\{p\left(c_{i}\right) \mid c_{i} \in C\right\}
$$

reflects the characteristics of the ambient population, the probability that two individuals $a$ and $b$, randomly chosen from an infinite population, would be found to belong to a particular class $c_{i}$ is:

$$
p\left(c_{i}\right) \times p\left(c_{i}\right)=p\left(c_{i}\right)^{2} .
$$

Since class membership is mutually exclusive, the probability that $a$ and $b$ would belong to the same class (irrespective of which particular class), is given by:

$$
\operatorname{Prob}(\operatorname{class}(a)=\operatorname{class}(b))=\sum_{c_{i} \in C} p\left(c_{i}\right)^{2}
$$

In the specific case of our data on New York City's methamphetamine-using population, the expression in Equation (2) evaluates to $6.21 \times 10^{-3}$. Multiplying this number by the probability that $a$ and $b$ will share the same telefunken code (1/64), yields the probability that two randomly chosen individuals will match by sheer chance:

$$
1 / 64 \times\left(6.21 \times 10^{-3}\right)=9.7 \times 10^{-5}
$$

Applying linearity of expectation, each specific participant expects:

$$
466 \times\left(9.7 \times 10^{-5}\right)=4.52 \times 10^{-2}
$$

reports (from among the 466) to match him/her by sheer chance. Linearity of expectation applied once more yields the total number of matches between the $(n=132)$ respondents and the $(s=466)$ reports that are attributable to sheer chance:

$$
F^{\prime \prime}=132 \times\left(4.52 \times 10^{-2}\right)=5.97 .
$$

The number $F^{\prime \prime}$ provides a more refined estimate of $E[F] \approx$ $F^{\prime \prime}$, since it takes into account the joint distribution of the ambient population from which the sample was drawn (to the extent that the distribution of attributes in the population conforms to that of the extended sample). Adjusting the recapture number $t^{\prime \prime}=t-F^{\prime \prime}$ to incorporate this more refined analysis of the expected false matches, yields $t^{\prime \prime}=11-5.97=5.03$, from which we derive the revised population estimate of $P^{\prime \prime}=$ 12,229 .

\section{Range of Estimates}

Developing a range of plausible population estimates requires moving beyond the study of expected values (i.e. $E[F]$ ), to acquire a deeper understanding of the probability distribution governing the number of false matches $F$. We begin by noting that $F$ represents the number of successes in a Bernoulli sequence of $132 \times 466=61,512$ trials-or 466 throws at 132 possible hits per throw-where the probability of success in any given trial is $9.7 \times 10^{-5}$ (see Equation (3)). The standard deviation of $F$ is thus given by a well-known fact concerning Bernoulli distributions:

$$
\operatorname{std}(F)=61,512 \times\left(9.7 \times 10^{-5}\right) \times\left(1-9.7 \times 10^{-5}\right) \approx 2.44
$$

This standard deviation can be used as a measure of the variability of $F$.

Population estimates based on the expected number of false matches should be seen as the midpoint of a range of estimates. Our estimate $F^{\prime \prime}$ can be better adjusted to incorporate this variability

$$
E[F] \approx F^{\prime \prime} \pm \operatorname{std}(F)=5.97 \pm 2.44 .
$$

The population estimate corresponding to $5.97+2.44=8.41$ false matches is:

$$
P^{+}=132 \times 466 /(11-8.41)=23,750 .
$$

while considering $5.97-2.44=3.53$ false matches yields: 


$$
P^{-}=132 \times 466 /(11-3.53)=8235 .
$$

By considering one standard deviation of the random variable $F$ around its estimated mean, we obtain a range of population estimates [8235, 23,750].

\section{Confidence Intervals}

To obtain confidence intervals for population estimates we use the Chernoff bound for the upper and lower tail of the distribution:

$$
\begin{aligned}
& \operatorname{Pr}(F>(1+\delta) E[F]) \leq\left(\frac{e^{\delta}}{(1+\delta)^{(1+\delta)}}\right)^{E[F]} . \\
& \operatorname{Pr}(F<(1-\delta) E[F]) \leq\left(\frac{e^{-\delta}}{(1-\delta)^{(1-\delta)}}\right)^{E[F]} .
\end{aligned}
$$

Using the previous $F^{\prime \prime}$ estimate of $E[F]$, the upper and lower bounds corresponding to these two equations are listed in the Table 3. As is evident from the table, one needs to expand to fairly wide estimates around $F^{\prime \prime}$ in order for the upper and lower bound confidence values to equalize, e.g., by considering the number of false matches $\mathrm{F}$ to lie between $3(60 \%)$ and 9 (49\%). This analysis indicates considerable sensitivity to false match frequencies, a result that is perhaps not surprising given the value of $\operatorname{std}(F)$.

As such, the $P^{\prime \prime}=12,229$ estimate based on $F^{\prime \prime}=5.97$ should be taken as a central value with a fairly wide range, with the understanding that the actual population size could be as high as 30,756 (if there were 9 false matches among the 11), or as low as 7689 (if there were only 3 false matches among the 11).

\section{Discussion}

Perhaps more interesting than the actual methamphetamine-using population estimates themselves, however, is the

Table 3.

Population estimates by confidence intervals.

\begin{tabular}{ccccc}
\hline $\begin{array}{c}\text { Upper bound } \\
\text { on false } \\
\text { matches }(k)\end{array}$ & $\begin{array}{c}\text { Upper bound } \\
\text { on Prob. } \\
(F<k)\end{array}$ & $\begin{array}{c}\text { Lower } \\
\text { bound on } \# \\
\text { of true } \\
\text { matches }\end{array}$ & $\begin{array}{c}\text { Lower } \\
\text { bound on } \\
\text { population } \\
\text { size }\end{array}$ & $\begin{array}{c}\text { Bound } \\
\text { confidence }\end{array}$ \\
\hline 1 & 0.04 & 10 & 6151 & 0.96 \\
2 & 0.17 & 9 & 6835 & 0.83 \\
3 & 0.40 & 8 & 7689 & 0.60 \\
4 & 0.69 & 7 & 8787 & 0.31 \\
5 & 0.92 & 6 & 10,252 & 0.08 \\
\hline $\begin{array}{c}\text { Lower } \\
\text { bound on } \\
\text { false }\end{array}$ & $\begin{array}{c}\text { Upper bound } \\
\text { on Prob. }\end{array}$ & $\begin{array}{c}\text { Upper bound } \\
\text { on } \# \text { of true } \\
\text { matches }\end{array}$ & $\begin{array}{c}\text { Upper bound } \\
\text { on popula- } \\
\text { tion size }\end{array}$ & Bound \\
\hline 7 & $(F)$ & confidence & \\
\hline 8 & 0.92 & 4 & 15,378 & 0.08 \\
9 & 0.73 & 3 & 20,504 & 0.27 \\
10 & 0.51 & 2 & 30,756 & 0.49 \\
\hline
\end{tabular}

estimation method. Capture-recapture techniques have retained an important place in socio-medical studies (e.g. Chao et al., 2001; Kruse et al., 2003; Hall et al., 2006; Vuylsteke, 2010), despite acknowledgment of long standing limitations (Hook \& Regal, 1995). Few of these methods have involved social network data, however, with recent network attention focused on scale-up methods, as discussed by Kadushin et al., (2006), McCormick et al., (2010) and Bernard et al., (2010). The method discussed in this paper is not a substitute for large scale estimation of the sort addressed by scale-up methods, but it does take steps toward alleviating the largest problems associated with traditional capture-recapture techniques: the need for two distinct samplings of the population (see Laska \& Meisner, 1993 for discussion), and the need for subject anonymity throughout the matching process when dealing with illegal or highly stigmatized behaviors (see Hook \& Regal, 1995). Because our method depends on data captured during a single survey and involves (what we feel to be) a reliable way to recognize matches while maintaining anonymity, as well as means for estimating the number of false matches, it addresses traditional problems associated with capture-recapture techniques for population estimates of illegal drug users.

We note, however, that the method described here assumes that the researcher has access to the hidden population, though not complete access, and that this access is capable of producing a representative sample ${ }^{5}$. The latter is perhaps the most problematic of these assumptions, and we recognize the difficulty of establishing, rather than simply assuming representativeness. Nevertheless, where population estimates of specific local subpopulations are sought, the method described here avoids complex issues such as determination of degree distributions of the population from which contact information is gathered, so-called "transmission errors", barrier effects, and recall error (as discussed by McCormick et al., 2010).

Obvious limitations contextualize these results. The most obvious of these is the representativeness of the sample to the larger population from which it is drawn, which is a fundamental assumption for both estimates, and one that rests on shaky ground. This was a small sample by RDS standards, and as such it is very likely that sample equilibrium had not been reached, and that sample skewing as a result of seed selection, volunteerism, and other peer-driven pitfalls affected the representativeness of the 132 recruits, and perhaps the 466 reports as well (the latter is equally important because the reports were used to estimate the space of variability of the ambient population in the second estimate as well). RDS recruitment methods also generally tend to enroll higher-than-representative numbers of well-connected individuals, simply by virtue of the fact that they have more chances to be recruited, which could skew the results should the ego-networks of these well-connected individuals differ from those of the remainder of the population in significant ways, i.e. ways that affect the demographics of the sample connections (see Berchenko \& Frost, 2011). And fi-

\footnotetext{
${ }^{5}$ Ideally, one would like to begin the matching procedure from a random sample of the population of interest. As has been clear from the beginning of the paper, however, the method proposed here is intended for situations where this is not possible. Inevitably, this means that we begin with something that is less than a random sample, but something more than a simple convenience sample (as the RDS method does provide some semblance of a random walk in the referral process, and means to estimate the limits of that randomness). As no current alternative exists for this situation, this remains an explicit and acknowledged limit of the method here, but one for which we currently do not have any alternative.
} 
nally, the use of peer referrals forces us to wonder whether the number matches discovered here $(t=11)$ were a result of the fact that recruits were drawn from a closely connected segments of the larger population, leading to a greater likelihood that individuals knew one another by virtue of being part of the same social clique (and thus lowering the estimated population figure). Given that both estimates assumed that the respondents had been chosen randomly from the population, such considerations cast doubt on the validity of the final estimate, which is likely larger than the figures given here ${ }^{6}$.

Nevertheless, the methods described here are in no way dependent on RDS as a method of recruitment, and may in fact be better suited to other methods (venue-based sampling or other techniques used to recruit hard-to-reach populations). In such cases, the likelihood that matches are the result of over-recruitment among a quasi-clique of well-connected respondents remains an open question as well. Still, with the growing popularity of mobile phones all over the world, the possibility of telefunken encoding as a means of anonymously matching network alters is rapidly expanding. In that case, the anonymized identification method of encoding phone numbers (even/odd, 0-4/5-9) as unique identifiers can potentially remedy one of the more difficult questions about how to expand ego-network data to larger chains of sociometric connection. As such, there may be potential for the extension of this method to other hard-to-reach populations, or to any population where network connections are a concern but where the solicitation of connection via name is not possible. Perhaps as importantly, this technique has the special virtue of deriving an estimate while retaining respondent anonymity and the anonymity of network alters, a frequent requirement of human subject protection and a common difficulty in attempting to link ego-data information gained in individual interviews into a larger network whole.

\section{Acknowledgements}

This project was supported by Award No. 2007-NIJ-CX0110 from the National Institute of Justice, Office of Justice Programs, U.S. Department of Justice. The opinions, findings, and conclusions or recommendations expressed in this publication are those of the authors and do not necessarily reflect those of the U.S. Department of Justice. See Wendel et al., 2011 for an expanded discussion of the research project from which the data for this analysis were taken.

\section{REFERENCES}

Aceijas, C., Friedman, S. R., Cooper H. L., Wiessing, L., Stimson, G. V., \& Hickman, M. (2006). Estimates of injecting drug users at the national and local level in developing and transitional countries, and gender and age distribution. Sexually Transmitted Infections, 82, iii10-iii17. doi:10.1136/sti.2005.019471

${ }^{6}$ Recent assessments (Gile \& Handcock, 2010; Goel \& Salganik, 2010) have found that RDS occasionally performs worse than expected. In particular, RDS Analysis Tool generated confidence interval estimates may be too small, and design effects of $5-10$ may be more likely than the previous assumed value of 2 . Both large design effects and incorrect confidence intervals occur when the underlying network has significant bottlenecks. In the example discussed here, we note that the overall size of the sample $(\mathrm{n}=$ 132 ) is not large enough to fulfill either the older (2), or the more recent (5 10) design effect limits. As stated below, this method of estimating population based on network sampling is not dependent on RDS recruiting methodologies and may even be hindered by them.
Arrestee Drug Abuse Monitoring (2009). ADAM II: 2009 Annual Report. Washington DC: US Office of National Drug Control Policy, Executive Office of the President.

http://www.whitehousedrugpolicy.gov/publications/pdf/adam2009.p df

Berchenko, Y., \& Frost, S. D. (2011) Editorial: Capture-recapture methods and respondent-driven sampling: Their potential and limitations. Sexually Transmitted Infections, 87, 267-268. doi:10.1136/sti.2011.049171

Bernard, H. R., Hallett, T., Iovita, A., Johnsen, E. C., Lyerla, R. McCarty, C., Mahy, M., Salganik, M. J., Saliuk, T., Scutelniciuc, O., Shelley, G. A., Sirinirund, P., Weir, S., \& Stroup, D. F. (2010). Counting hard-to-count populations: The network scale-up method for public health. Sexually Transmitted Infections, 86, ii11-ii15. doi:10.1136/sti.2010.044446

Bouchard, M. (2007). A capture-recapture model to estimate the size of criminal populations and the risks of detection in a marijuana cultivation industry. Journal of Quantitative Criminology, 23, 221-241. doi: 10.1007/s10940-007-9027-1

Chao, A., Tsay, P. K., Lin, S. H., Shau, W. Y., \& Chao, D. Y. (2001). The applications of capture-recapture models to epidemiological data. Statistics in Medicine, 20, 3123-3157.

Degenhardt, L., \& Hall, W. (2012). Extent of illicit drug use and dependence, and their contribution to the global burden of disease. Lancet, 379, 55-70. doi:10.1016/S0140-6736(11)61138-0

Drug Abuse Warning Network (2009). National estimates of drugrelated emergency department visits, 2004-2008, illicit drug visits. Washington DC: Substance Abuse and Mental Health Services Administration, US Department of Health and Human Services. https://dawninfo.samhsa.gov/data/report.asp?f=Nation/Illicit/Nation 2008_Illicit_ED_Visits_by_Drug

Drug Abuse Warning Network (2010). Emergency department visits involving methamphetamine: 2004-2008. Washington DC: Substance Abuse and Mental Health Services Administration, US Department of Health and Human Services.

https://dawninfo.samhsa.gov/files/SpecTopics/DAWN2010SR017.pd $\mathrm{f}$

Drug Enforcement Administration (2004). US charges New York crystal meth dealer ring. URL (last checked 2 March 2004). https://www.dea.gov/pubs/states/newsrel/nyc030204.html

Drug Enforcement Administration (2006). Meth in the city: 9 meth labs found, 10 charged in New York City and Long Island. URL (last checked 30 November 2006).

https://www.dea.gov/pubs/states/newsrel/nyc113006.html

Gile, K. J., \& Handcock, M. S. (2010). Respondent-driven sampling: An assessment of current methodology. Sociological Methodology, 40, 285-327. doi: 10.1111/j.1467-9531.2010.01223.x

Gile, K. J., Johnston, L. G., \& Salganik, M. J. (2012). Diagnostics for respondent driven sampling. arXiv: $1209.6254 \mathrm{v} 1$

Goel, S., \& Salganik M. J. (2010). Assessing respondent-driven sampling. Proceedings of the National Academy of Sciences, 107, 67436747. doi:10.1073/pnas.1000261107

Hall, H. I., Song, R., Gerstle III, J. E., \& Lee L. M. (2006). Assessing the completeness of reporting of Human Immunodeficiency Virus diagnoses in 2002-2003: Capture-recapture methods. American Journal of Epidemiology, 164, 391-397.

Heckathorn, D. (1997). Respondent-driven sampling: A new approach to the study of hidden populations. Social Problems, 44, 174-199. doi: $10.2307 / 3096941$

Heckathorn, D. (2002). Respondent-driven sampling II: Deriving valid population estimates from chain-referral samples of hidden populations. Social Problems, 39, 11-34. doi:10.1525/sp.2002.49.1.11

Heckathorn, D. (2007). Extensions of respondent-driven sampling: Analyzing continuous variables and controlling for differential recruitment. Sociological Methodology, 37, 151-208. doi:10.1111/j.1467-9531.2007.00188.x

Hirshfield, S., Remien, R., Walavalkar, I., \& Chiasson, M. (2004). Crystal methamphetamine use predicts incident STD infection among men who have sex with men recruited online: A nested case-control study. Journal of Medical Internet Research, 6, e41. doi:10.2196/jmir.6.4.e41 
Hope, V., Hickman, M., \& Tilling, K. (2005). Capturing crack cocaine use: Estimating the prevalence of crack cocaine use in London using capture-recapture with covariates. Addiction, 100, 1701-1708. doi: 10.1111/j.1360-0443.2005.01244.x

Hook, E. B., \& Regal, R. R. (1995). Capture-recapture methods in epidemiology: Methods and limitations. Epidemiology Review, 17, 243-264.

Johnston, L. G., Malekinejad, M., Kendall, C., Iuppa, I. M., \& Rutherford, G. W. (2008). Implementation challenges to using respondentdriven sampling methodology for HIV biological and behavioral surveillance: Field experiences in international settings. AIDS and Behavior, 12, 131-141.

Kadushin, C., Killworth, P. D., Bernard, H. R., \& Beveridge, A. A. (2006). Scale-up methods as applied to estimates of heroin use. Journal of Drug Issues, 36, 417-440. doi: $10.1177 / 002204260603600209$

Kruse, N., Behets, F., Vaovola, G., Burkhardt, G., Barivelo, T., Amida, X., \& Dallabetta, G. (2003). Participatory mapping of sex trade and enumeration of sex workers using capture-recapture methodology in Diego-Suarez, Madagascar. Sexually Transmitted Diseases, 30, 664670.

Laska, E. M., \& Meisner, M. A. (1993). A plant-capture method for estimating the size of a population from a single sample. Biometrics, 49, 209-220. http://www.jstor.org/stable/2532614

Maxwell, J., \& Rutkowski, B. (2008). The prevalence of methamphetamine and amphetamine abuse in North America: A review of the indicators, 1992-2007. Drug and Alcohol Review, 27, 229-235.

McCormick, T. H., Salganik, M. J., \& Zheng, T. (2010). How many people do you know? Efficiently estimating personal network size. Journal of the American Statistical Association, 105, 59-70. doi:10.1198/jasa.2009.ap08518

Morin, S., Steward, W., Charlebois, E., Remien, R., Pinkerton, S., Johnson, M., Rotheram-Borus, M., Lightfoot, M., Goldstein, R., Kittel, L., Samimy-Muzaffar, F., Weinhardt, L., Kelly, J., \& Chesney, M., (2005). Predicting HIV transmission risk among HIV-infected men who have sex with men: Findings from the healthy living pro- ject. Journal of Acquired Immune Deficiency Syndromes, 40, 226235.

National Drug Intelligence Center (2008). Methamphetamine Threat Assessment 2009. Washington DC: US Department of Justice.

Paz-Bailey, G., Jacobson, J. O., Guardado, M. E., Hernandez, F. M., Nieto, A. I., Estrada, M., \& Creswell, J. (2011). How many men who have sex with men and female sex workers live in El Salvador? Using respondent-driven sampling and capture-recapture to estimate population sizes. Sexually Transmitted Infections, 87, 279-282.

Salganik, M. J., Fazito, D., Bertoni, N., Abdo, A. H., Mello, M. B., \& Bastos, F. I. (2011). Assessing network scale-up estimates for groups most at risk of HIV/AIDS: Evidence from a multiple-method study of heavy drug users in Curitiba, Brazil. American Journal of Epidemiology, 174, 1190-1196. doi: 10.1093/aje/kwr246

Schoeneberger, M., Leukefeld, C., Hiller, M., \& Godlaski, T. (2006). Substance abuse among rural and very rural drug users at treatment entry. American Journal of Drug and Alcohol Abuse, 32, 87-110.

Simeone, R., Holland, L., \& Viveros-Aquilero, R. (2003). Estimating the size of an illicit-drug-using population. Statistics in Medicine, 22 2969-2993. doi: 10.1002/sim. 1528

Thoumi, T. (2005). The numbers game: Let's all guess the size of the illegal drug industry! Journal of Drug Issues, 35, 185-200. doi:10.1177/002204260503500109

Vuylsteke, B., Vandenhoudt, H., Langat, L., le Semde, G., Menten, J., Odongo, F., Anapapa, A., Sika, L., Buve, A., \& Laga, M. (2010). Capture-recapture for estimating the size of the female sex worker population in three cities in Cote d'Ivoire and in Kisumu, western Kenya. Tropical Medicine and International Health, 15, 1537-1543.

Wendel, T., Khan, B., Dombrowski, K., Curtis, R., McLean, K., Misshula, E., Riggs, R., \& Marshall IV, D. M. (2011). Dynamics of retail methamphetamine markets in New York City. Final report to the National Institute of Justice, office of justice programs. Washington DC: US Department of Justice.

Zhao, Y. (2011). Estimating the size of an injecting drug user population. World Journal of AIDS, 1, 88-93. doi: $10.4236 /$ wja. 2011.13013 